\title{
Cardiac metastasis in a patient with poorly-differentiated neuroendorcrine tumor: 18F-FDG PET-CT finding
}

\section{KEYWORDS: poorly differentiated NETs — 18F-FDG PET-CT a cardiac metastasis}

We describe 18F-FDG positon emission tomography-computed tomography findings of a 67 y old male patient, followed since 2014 for poorly differentiated NETs with unknown primary tumor. PET-CT showed cardiac hypermetabolism in the left ventricular as muscular metastasis in addition to other muscular metastases of the two diaphragmatic pillars and the left iliopsoas muscle (FIGURE 1). Cardiac USN and chest-CT (FIGURES $2 \&$ 3) confirmed our diagnostic by showing a small lesion in the cardiac apex measuring $13.7 \times 18$ $\mathrm{mm}$.
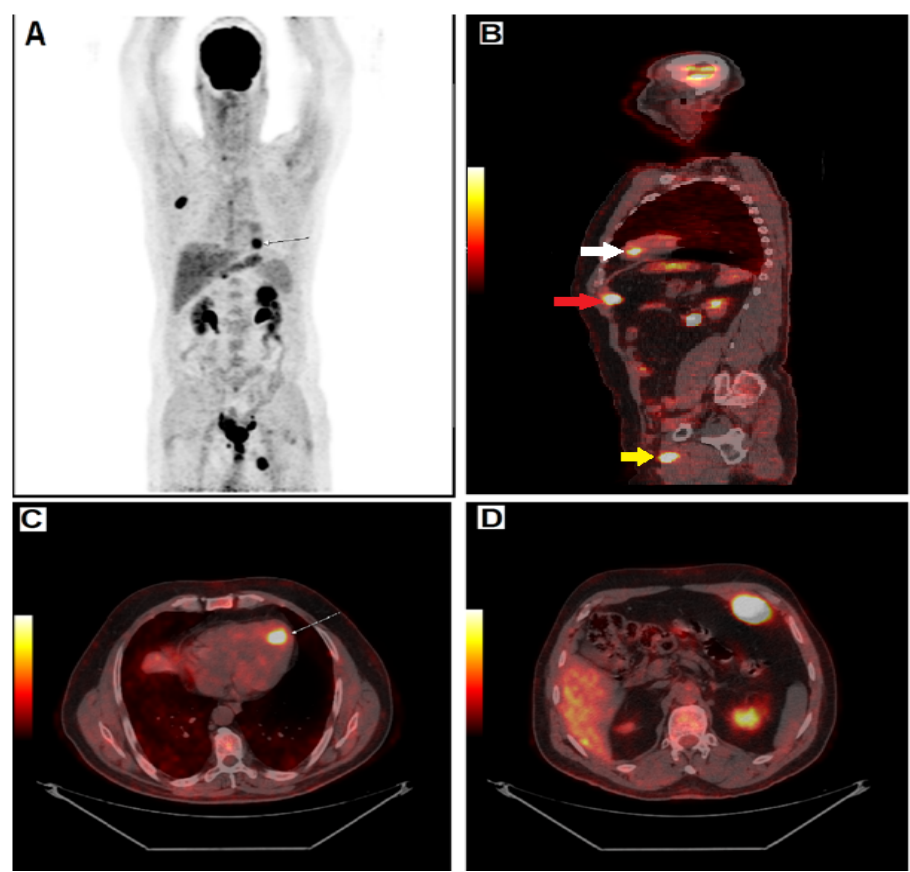

Figure 1. (A) Maximum intensity projection (MIP). (B) Sagittal sectional fusion images showing cardiac metastasis in the left ventricle (white arrow), muscular metastases of the two diaphragmatic pillars (red arrow) and the left iliopsoas muscle (yellow arrow). (C) Fusion image in axial section showing myocardial hypermetabolism (white arrow) in the cardiac apex (SUVmax =11.6). (D) Fusion image in axial section showing muscular hyper metabolism related to metastasis in the left diaphragmatic pillar, measuring 45.9 x $33.0 \mathrm{~mm}$ (SUVmax = 20.8).
Myocardial metastases are very rare with a reported incidence up to 1 to $4 \%$ [1]. 18F-FDG is a positron emission tomography tracer for poorly differentiated NETs with KI67>20\% [2]. This modality of exploration is useful for the staging and the restaging patients. The maximum detection was correlated to a low somatostatin receptor (SSRT) expression unlike well-differentiated tumors that show great expression of these receptors [3].

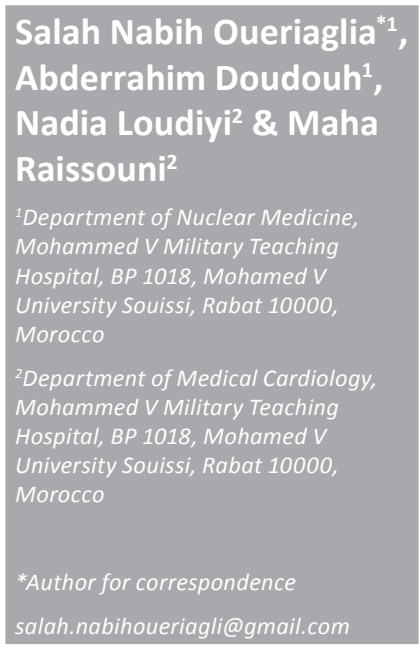




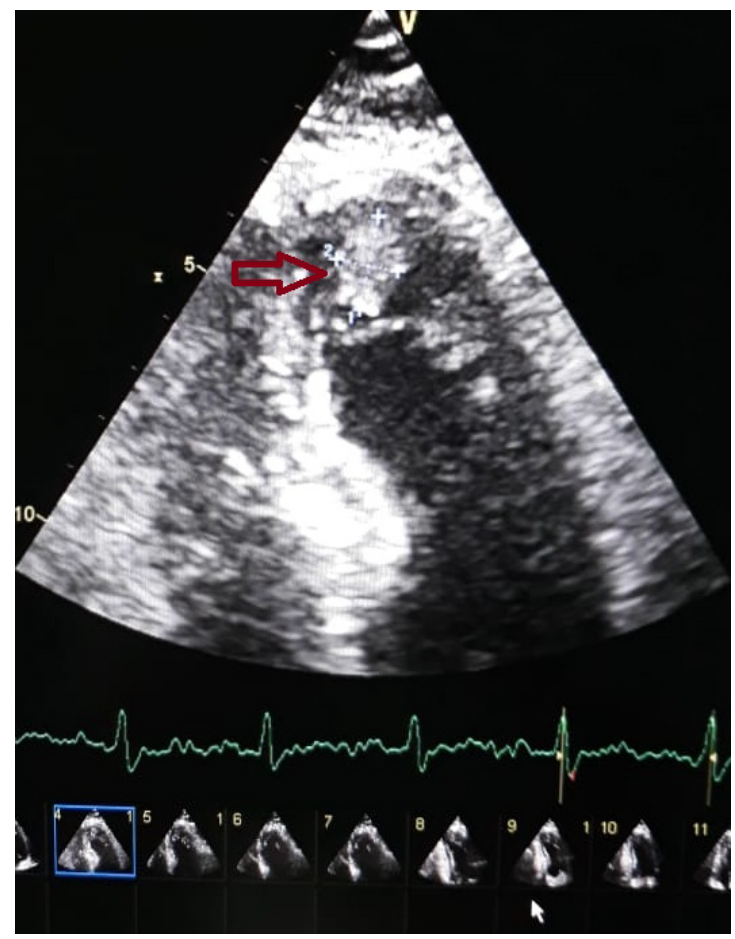

Figure 2. Cardiac ultrasonography showing multilobular mobile mass in the tip of the left ventricle measuring $13.7 \mathrm{~mm} \times 18 \mathrm{~mm}$ (red arrow).

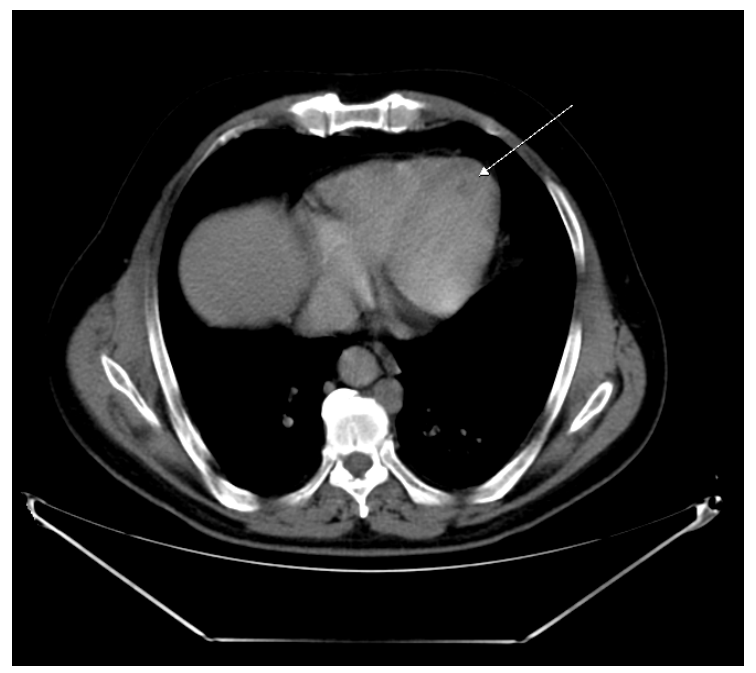

Figure 3. Chest CT showing a small hypodense lesion in the cardiac apex corresponding to the focal uptake demonstrated on PET-CT.

\section{REFERENCES}

1. Jann $H$, Wertenbruch $T$, Pape $U$ et al. A matter of the heart: myocardial metastases in neuroendocrine tumors. Horm. Metab. Res. 42: 967-976, (2010).
2. Koopmans KP, Neels ON, Kema IP et al. Molecular imaging in neuroendocrine tumors: Molecular uptake mechanisms and clinical results. Crit. Rev. Oncol. Hematol. 71: 199-213, (2009).
3. Khangembam BC, Naswa N, Sharma P et al. Isolated cardiac metastasis in a patient with neuroendocrine carcinoma of pancreas discovered on Ga-68-DOTANOC PET/CT. $J$. Nucl. Cardiol. 19: 1078-1079, (2012). 